\title{
CDISC Protocol Entities Physical Address Attribute Terminology
}

National Cancer Institute

\section{Source}

National Cancer Institute. CDISC Protocol Entities Physical Address Attribute

Terminology. NCl Thesaurus. Code C132308.

Terminology associated with the protocol entity physical address attribute codelist of the Clinical Data Interchange Standards Consortium (CDISC). 